\title{
Desafios e perspectivas para a administração segura de medicamentos pela Enfermagem
}

\author{
Challenges and perspectives for the safe administration of drugs by Nursing
}

Desafíos y perspectivas para la administración segura de medicamentos por la Enfermería

\begin{abstract}
Mardem Augusto Paiva Rocha Júnior ${ }^{1}$, Francisco Lucas de Lima Fontes ${ }^{1 *}$, Layany Feitosa Pinho ${ }^{1}$, Samuel Lopes dos Santos ${ }^{2}$, Ilana Maria Brasil do Espírito Santo ${ }^{3}$, Bruna Furtado Sena de Queiroz ${ }^{2}$, Mônika Cristina de Oliveira ${ }^{1}$, Emilene Paz Freitas ${ }^{4}$, Ayla Cristina Rodrigues Ramos da Costa ${ }^{1}$, Francisca Jéssica Abreu da Silva ${ }^{1}$, Maria Idalina Rodrigues ${ }^{5}$, Karine do Nascimento Miranda Martins Granjeiro $^{6}$, Brenda Lícia Martins da Silva1, Jadson Farias de Sousa ${ }^{1}$, Lídia Viana Araújo․
\end{abstract}

\section{RESUMO}

Objetivo: Identificar desafios e perspectivas para a administração segura de medicamentos pela Enfermagem. Métodos: Trata-se de um estudo descritivo, sedimentado na revisão integrativa de natureza exploratória. A busca dos estudos foi realizada na biblioteca virtual Scientific Eletronic Library Online (SciELO) e nas bases de dados Literatura Latino-Americana e do Caribe em Ciências da Saúde (LILACS) e Periódicos da CAPES. Resultados: Foram encontrados 367 artigos relevantes à temática e após critérios de inclusão e exclusão, selecionados 10 artigos para edificação deste estudo. Emergiu-se na discussão dois tópicos: desafios e perspectivas sobre a administração segura de medicamentos pela Enfermagem. Considerações finais: Os profissionais de Enfermagem enfrentam desafios como sobrecarga de trabalho, falhas associadas à estrutura física e material, condições do ambiente (desorganização) e interrupções frequentes ao serem responsáveis por muitos procedimentos e pacientes. A capacitação e treinamentos podem ser estratégias viáveis à mudança desse cenário problemático, estimulando o profissional de Enfermagem à realização de boas práticas na administração de medicamentos.

Palavras-chave: Segurança do paciente, Gerenciamento de segurança, Sistemas de medicação, Cuidados de Enfermagem.

\footnotetext{
ABSTRACT

Objective: To identify challenges and perspectives for the safe administration of medications by Nursing. Methods: This is a descriptive study, consolidated in the integrative review of an exploratory nature. The search for the studies was carried out in the Scientific Eletronic Library Online (SciELO) virtual library in the Latin American and Caribbean Literature in Health Sciences (LILACS) and CAPES Periodicals databases. Results: We found 367 articles relevant to the theme, and after inclusion and exclusion criteria, we selected 10 articles to build this study. Two topics emerged in the discussion: challenges and perspectives on the safe administration of drugs by Nursing. Final considerations: Nursing professionals face challenges such as work overload, failures associated with physical and material structure, environmental conditions (disorganization),

${ }^{1}$ Faculdade UNINASSAU - Campus Redenção. Teresina, Piauí, Brasil. *E-mail: lucasfontesenf@hotmail.com

${ }^{2}$ Faculdade FACID - Wyden. Teresina, Piauí, Brasil.

${ }^{3}$ Centro Universitário Uninovafapi. Teresina, Piauí, Brasil.

${ }^{4}$ Centro Universitário Santo Agostinho. Teresina, Piauí, Brasil.

${ }^{5}$ Faculdade UNINASSAU. São Luís, Maranhão, Brasil.

${ }^{6}$ Universidade do Rio Grande. Rio de Janeiro, Rio de Janeiro, Brasil.
}

SUBMETIDO EM: 2/2019

ACEITO EM: 3/2019

PUBLICADO EM: 6/2019

REAS/EJCH | Vol.Sup.25 | e452 | DOI: https://doi.org/10.25248/reas.e452.2019 Página 1 de 8 
and frequent disruptions in being responsible for many procedures and patients. Training and training can be viable strategies to change this problematic scenario, stimulating the Nursing professional to perform good practices in drug administration.

Keywords: Patient safety, Security management, Medication systems, Nursing care.

\section{RESUMEN}

Objetivo: Identificar desafíos y perspectivas para la administración segura de medicamentos por la Enfermería. Métodos: Se trata de un estudio descriptivo, sedimentado en la revisión integrativa de naturaleza exploratoria. La investigación de los estudios se realizó en la biblioteca virtual Scientific Electronic Library Online (SciELO) y en las bases de datos Literatura Latinoamericana y del Caribe en Ciencias de la Salud (LILACS) y Periódicos de la CAPES. Resultados: Se han encontrado 367 artículos relevantes a la temática, y después de criterios de inclusión y exclusión, seleccionados 10 artículos para edificación de este estudio. Se planteó en la discusión dos temas: desafíos y perspectivas sobre la administración segura de medicamentos por la enfermería. Consideraciones finales: Los profesionales de enfermería enfrentan desafíos como sobrecarga de trabajo, fallas asociadas a la estructura física y material, condiciones del ambiente (desorganización) e interrupciones frecuentes al ser responsables por muchos procedimientos y pacientes. La capacitación y entrenamientos pueden ser estrategias viables al cambio de ese escenario problemático, estimulando al profesional de Enfermería a la realización de buenas prácticas en la administración de medicamentos.

Palabras-clave: Seguridad del paciente, Gestión de la seguridad, Sistemas de medicación, Atención de Enfermería.

\section{INTRODUÇÃO}

A segurança do paciente trata-se do ato de evitar, prevenir ou reduzir a ocorrência dos eventos adversos ou lesões geradas nos serviços de saúde em geral. A ausência de protocolos ocasiona 42,7 milhões de eventos adversos mundialmente, destes, dois terços ocorrem em países menos desenvolvidos e de transição. Em países desenvolvidos, um em cada dez pacientes é prejudicado ao receber cuidados hospitalares. Apesar do cuidado em saúde ser realizado visando benefícios ao paciente, a ocorrência de erros é possível, e os pacientes podem sofrer graves consequências (JHA AK et al., 2013; SILVA ACA et al., 2016).

Depois da divulgação do relatório do Institute of Medicine chamado To Err is Human: building a safer health system, nos Estados Unidos, o tema segurança do paciente ganhou relevância mundial. O relatório estimou que cerca de 44.000 a 98.000 pessoas morrem anualmente devido a erros médicos evitáveis. (KOHN LT et al., 2000). No Brasil, de 2014 a 2016 foram notificados 63.933 eventos adversos associados à assistência à saúde. Desses casos, 417 tiveram como desfecho o óbito (MAIA CS et al., 2018).

O erro é entendido como uma circunstância que poderia ter resultado ou resultou em dano desnecessário ao paciente, procedente ou não de atos intencionais. Um erro de medicação é incidente evitável que ocorre em qualquer etapa do processamento. Quando não alcança o paciente ou é detectado antes, pode ser denominado de quase erro ou near miss, um incidente que, por algum motivo, planejado ou pelo acaso, foi interrompido antes de atingir o paciente e poderia ou não causar danos (DUARTE SCM et al., 2015).

A mídia brasileira, não raramente, divulga casos nos quais houve prejuízos à saúde dos pacientes por erros, omissão de socorro, negligência, imprudência e imperícia de profissionais de saúde. De acordo com o Anuário da Segurança Assistencial Hospitalar no Brasil, aproximadamente 829 brasileiros vão a óbito diariamente em serviços hospitalares públicos de privados, o que corresponde a três mortes por falhas na assistência a cada cinco minutos (COUTO RC et al., 2017). 
A identificação de incidentes é fundamental e existem esforços para superar o problema da subnotificação com o desenvolvimento de sistemas informatizados que estão cada vez mais em uso pelos gestores nas unidades de saúde. Os maiores incidentes que ocorrem com o paciente relacionam-se a erros diagnósticos; falhas no processo de medicação; e práticas negligentes, imprudentes e de imperícia. A ocorrência desses eventos repercute na família e reduz a confiança do paciente no sistema de saúde, sem mencionar as consequências financeiras que acarreta (LEUFER T e CLEARY-HOLDFORTH J, 2013; CAPUCHO HC et al., 2013).

As estratégias para o fortalecimento de práticas seguras têm sido prioridade nas agendas das instituições, organizações e sistemas de saúde. Sobre o tema ocorreu crescimento de mais $100 \%$ no número de publicações sobre a temática em 2013, comparando ao ano de 2009. No Brasil, a segurança do paciente está pautada pela Portaria no 529 do Ministério da Saúde, que fundou o Programa Nacional de Segurança do Paciente (PNSP), e pela Resolução no 36 da Diretoria Colegiada (RDC)/Anvisa, que estabeleceu estratégias a fim da promoção da segurança do paciente e a melhoria da qualidade nos serviços de saúde no país. A Resolução no 36 de 2013 reforçou a utilização de protocolos, como o Protocolo de Segurança na Prescrição, Uso e Administração de Medicamentos, como estratégia organizacional de promoção do cuidado seguro (LIMA JÚNIOR AJ e GERHARDT LM, 2017).

Os hospitais precisam de mais suporte e treinamento quando o assunto é a discussão de erros na administração de medicamentos. Assim, cabe a essas instituições operacionalizar um sistema sobre erros, discuti-lo, investir em programas, e oferecer suporte emocional tanto para o paciente quanto para os profissionais envolvidos nesses eventos (MCLENNAN SR et al., 2016).

Os deslizes ou erros mais comuns de administração de medicamentos podem estar relacionados com atos inseguros seguidos por carência do conhecimento e erros na execução de protocolo. Condições que provocavam erros na administração de medicamentos incluem: comunicação inadequada (prescrição, documentação, transcrição da medicação), problemas com abastecimento de medicamentos e armazenamento (farmácia), erros no gerenciamento de fármacos, problemas relacionados a fadiga e o estresse no ambiente de trabalho durante a administração de medicamento (KEERS RN et al., 2013).

A Enfermagem é responsável pela etapa final na administração de medicamentos. Falhas cometidas ao longo do processo de administração nem sempre são percebidas, o que exige compromisso da categoria na correção de erros ocorridos anteriormente, prevenindo e garantindo a segurança do paciente (FASSARELLA CS et al., 2013).

Nesse sentido, torna-se relevante analisar na literatura a temática segurança do paciente nas instituições de saúde e a partir dessa reflexão ressaltar o papel da Enfermagem, com garantia de segurança em todo o processo de administração medicamentosa ao paciente. Este estudo poderá oferecer subsídios para prevenção desses eventos e para a implementação de um processo sistematizado, que garanta a segurança do paciente. Diante do que foi explanado, este estudo teve como objetivo identificar desafios e perspectivas para a administração segura de medicamentos pela Enfermagem.

\section{MÉTODOS}

Trata-se de um estudo descritivo do tipo revisão integrativa da literatura de natureza exploratória. A revisão integrativa apresentou notável penetração na área da Enfermagem na última década. Esse fato pode estar associado à tendência de compreender o cuidado em saúde como um trabalho complexo que requer colaboração e integração de conhecimentos de diversas áreas. Esta metodologia permite incluir estudos experimentais e não-experimentais, de forma que auxiliem para uma compreensão completa do fenômeno analisado, além de combinar dados da literatura teórica e empírica (SOARES CB, et al., 2014).

Para sua confecção seguiu-se seis fases: elaboração da pergunta norteadora, busca ou amostragem na literatura, coleta de dados, análise crítica dos estudos incluídos, discussão dos resultados e apresentação da revisão integrativa (SOUZA MT, et al., 2010). A questão norteadora foi elaborada por meio da estratégia PICO 
(P: paciente ou problema, I: intervenção, C: comparação e O: outcomes ou desfecho) conforme segue: "Quais desafios e perspectivas para a administração segura de medicamentos pela Enfermagem?".

A pesquisa foi realizada nos meses de setembro e outubro de 2018 utilizando como critérios de inclusão artigos primários, disponíveis na íntegra, de maneira gratuita, em língua portuguesa, publicados entre 2013 e 2017. Como critérios de exclusão optou-se por não utilizar livros, monografias, dissertações e teses, resumos de anais de eventos, artigos duplicados, em outros idiomas e que não estivessem relacionados à temática estudada.

O levantamento dos artigos na literatura foi realizado por meio de pesquisa na biblioteca virtual Scientific Eletronic Library Online (SciELO) e nas bases de dados Literatura Latino-Americana e do Caribe em Ciências da Saúde (LILACS) e Periódicos da CAPES.

Durante a busca, os descritores foram cruzados entre si com o uso do operador booleano "and", empregouse também o filtro de datas em todas as buscas. Foram utilizados, para busca dos artigos os seguintes descritores: "erro de medicação", "erros de Enfermagem", "humanização", "segurança do paciente" e "iatrogenia".

\section{RESULTADOS}

Depois de percorrida a trajetória metodológica para busca dos estudos nas fontes de dados (Figura 1), foi elaborado um instrumento para coleta das informações, em que foram coletadas as seguintes variáveis: autor/ano, objetivo, métodos e resultados. Tanto a análise quanto a síntese dos dados extraídos dos artigos que foram realizadas de forma detalhada, possibilitando examinar, relatar e organizar os dados, com intuito de reunir o conhecimento produzido sobre o tema explorado na revisão.

Figura 1 - Percurso metodológico para busca nas bases de dados.

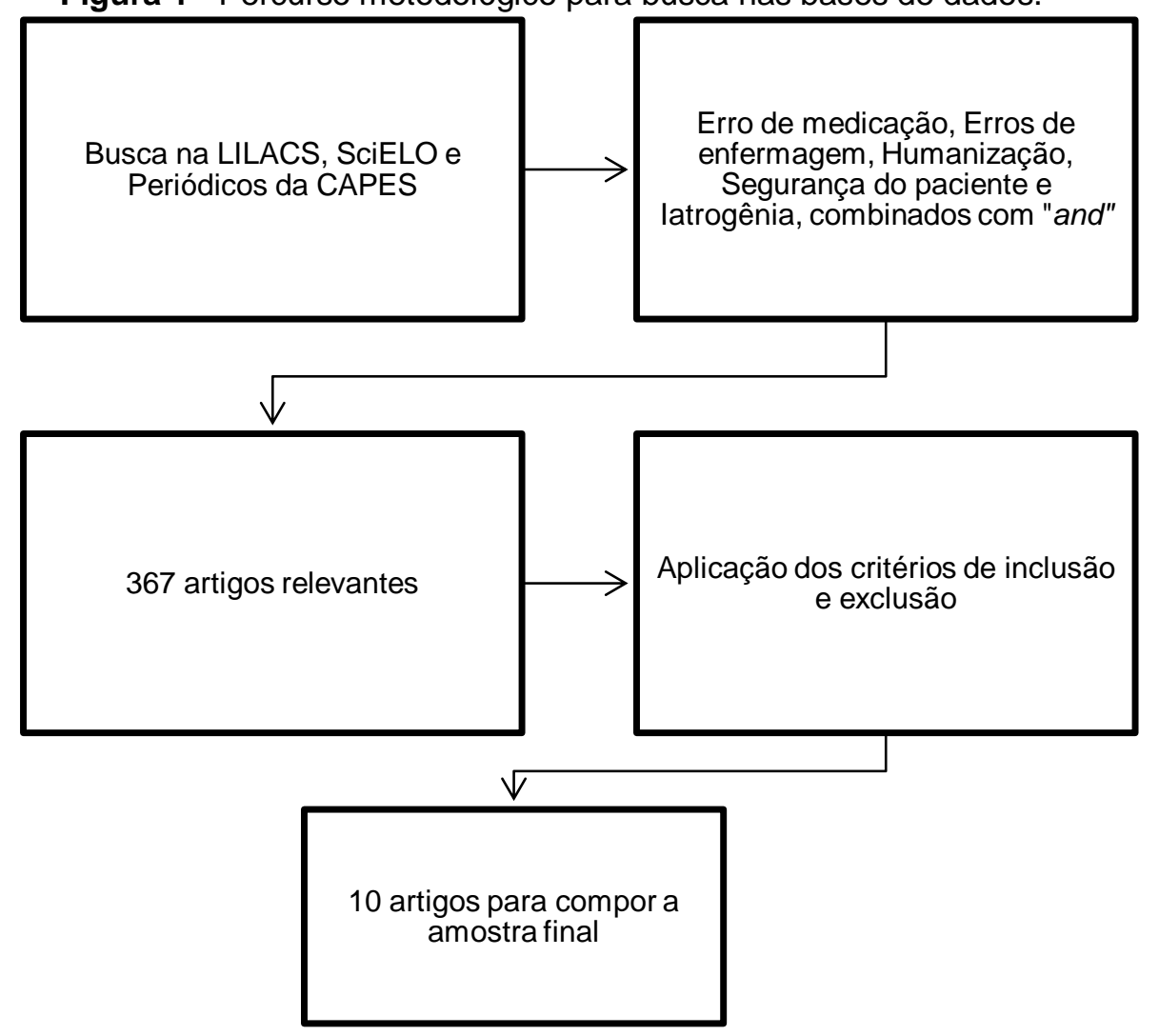

Fonte: Dados da pesquisa, 2018. 
De acordo com o delineamento da pesquisa, foi elaborado um quadro com a distribuição do autor/ano, objetivo do estudo, o método utilizado e os principais resultados dos estudos (Quadro 1).

Quadro 1 - Artigos selecionados de acordo com autor/ano, objetivo, metodologia aplicada e resultado encontrado no estudo. Teresina, Piauí, Brasil, 2018.

\begin{tabular}{|c|c|c|c|}
\hline AUTOR/ANO & OBJETIVO & MÉTODO & RESULTADOS \\
\hline $\begin{array}{l}\text { CHERNICHARO IM } \\
\text { et al., } 2013\end{array}$ & $\begin{array}{l}\text { Identificar os significados atribuídos } \\
\text { por profissionais de enfermagem aos } \\
\text { termos humanização e não } \\
\text { humanização. }\end{array}$ & $\begin{array}{c}\text { Estudo } \\
\text { qualitativo }\end{array}$ & $\begin{array}{l}\text { A assistência de Enfermagem vai ao } \\
\text { encontro dos significados dos próprios } \\
\text { partícipes do cuidado, devendo, } \\
\text { portanto, ser considerada como } \\
\text { coautora no processo saúde-doença. }\end{array}$ \\
\hline REIS LS et al., 2013 & $\begin{array}{l}\text { Identificar a percepção da equipe de } \\
\text { Enfermagem sobre a humanização no } \\
\text { cuidado em uma unidade de terapia } \\
\text { intensiva neonatal e pediátrica. }\end{array}$ & $\begin{array}{l}\text { Estudo } \\
\text { qualitativo }\end{array}$ & $\begin{array}{l}\text { Identificou-se que a compreensão da } \\
\text { equipe de enfermagem sobre } \\
\text { humanização pauta-se na própria } \\
\text { ciência do cuidado de Enfermagem, e } \\
\text { não especificamente na Política } \\
\text { Nacional de Humanização. }\end{array}$ \\
\hline $\begin{array}{l}\text { SILVA AEBC e } \\
\text { CASSIANI SHB, } \\
2013\end{array}$ & $\begin{array}{l}\text { Analisar os riscos potenciais do } \\
\text { processo de administração de } \\
\text { medicamentos anti-infecciosos por via } \\
\text { intravenosa. }\end{array}$ & $\begin{array}{c}\text { Estudo } \\
\text { quantitativo }\end{array}$ & $\begin{array}{l}\text { As causas das falhas relacionaram-se à } \\
\text { gestão dos processos organizacionais, } \\
\text { recursos humanos, estrutura física e } \\
\text { material. }\end{array}$ \\
\hline $\begin{array}{l}\text { NOVARETTI MCZ } \\
\text { et al., } 2014\end{array}$ & $\begin{array}{l}\text { Identificar a influência da sobrecarga } \\
\text { de trabalho da Enfermagem na } \\
\text { ocorrência de incidentes sem lesão e } \\
\text { eventos adversos. }\end{array}$ & $\begin{array}{c}\text { Estudo } \\
\text { quantitativo }\end{array}$ & $\begin{array}{l}\text { A ocorrência de incidentes foi atribuída à } \\
\text { sobrecarga de trabalho, o que elevou o } \\
\text { número de dias de internação e o risco } \\
\text { de óbito dos pacientes estudados. }\end{array}$ \\
\hline $\begin{array}{l}\text { DUARTE SCM et } \\
\text { al., } 2015\end{array}$ & $\begin{array}{l}\text { Identificar os erros no cotidiano da } \\
\text { assistência de Enfermagem em terapia } \\
\text { intensiva. }\end{array}$ & $\begin{array}{l}\text { Estudo } \\
\text { qualitativo }\end{array}$ & $\begin{array}{l}\text { O erro humano na assistência de } \\
\text { Enfermagem pode ser relacionado à } \\
\text { abordagem do sistema, através das } \\
\text { falhas ativas e condições latentes. }\end{array}$ \\
\hline $\begin{array}{c}\text { MAGALHÃES AMM } \\
\text { et al., } 2015\end{array}$ & $\begin{array}{l}\text { Levantar pontos críticos do processo } \\
\text { de medicação e suas repercussões } \\
\text { nas demandas de trabalho da equipe } \\
\text { de Enfermagem. }\end{array}$ & $\begin{array}{l}\text { Estudo } \\
\text { qualitativo }\end{array}$ & $\begin{array}{l}\text { O processo de medicação assume um } \\
\text { caráter de centralidade na organização } \\
\text { do trabalho da equipe de Enfermagem. }\end{array}$ \\
\hline $\begin{array}{l}\text { MARINI DC et al., } \\
2016\end{array}$ & $\begin{array}{l}\text { Identificar a frequência de erros } \\
\text { ocorridos no processo de diluição de } \\
\text { medicamentos intravenosos. }\end{array}$ & $\begin{array}{c}\text { Estudo } \\
\text { quantitativo. }\end{array}$ & $\begin{array}{l}\text { Os profissionais envolvidos com os erros } \\
\text { de medicação não souberam identificá- } \\
\text { los, e este tipo de evento pode } \\
\text { comprometer a eficácia terapêutica } \\
\text { medicamentosa. }\end{array}$ \\
\hline $\begin{array}{l}\text { HOEFEL HHK et al., } \\
2017\end{array}$ & $\begin{array}{l}\text { Analisar os incidentes de segurança } \\
\text { ocorridos com os pacientes durante o } \\
\text { cuidado de enfermagem. }\end{array}$ & $\begin{array}{c}\text { Estudo } \\
\text { quantitativo }\end{array}$ & $\begin{array}{l}\text { Os incidentes relacionados a } \\
\text { procedimentos técnicos contribuíram } \\
\text { com } 82 \% \text { ocorrências, sendo que em } \\
57 \% \text { ocorreram eventos adversos. }\end{array}$ \\
\hline $\begin{array}{l}\text { MOREIRA APA et } \\
\text { al., } 2017\end{array}$ & $\begin{array}{l}\text { Identificar quais são as dificuldades da } \\
\text { equipe de Enfermagem no manejo das } \\
\text { tecnologias durante a terapia } \\
\text { intravenosa (TIV). }\end{array}$ & $\begin{array}{l}\text { Estudo } \\
\text { qualitativo }\end{array}$ & $\begin{array}{l}\text { A maior dificuldade de ênfase cognitiva e } \\
\text { técnica foi a falta de treinamento; e de } \\
\text { ênfase administrativa, foi a falta de } \\
\text { recursos materiais e humanos. }\end{array}$ \\
\hline $\begin{array}{l}\text { TOMAZONI A et al., } \\
2017\end{array}$ & $\begin{array}{l}\text { Descrever a segurança do paciente na } \\
\text { percepção dos profissionais de } \\
\text { enfermagem e medicina de Unidades } \\
\text { de Terapia Intensiva Neonatal. }\end{array}$ & $\begin{array}{l}\text { Estudo } \\
\text { qualitativo }\end{array}$ & $\begin{array}{l}\text { Os profissionais refletem a necessidade } \\
\text { de incentivo para a comunicação de } \\
\text { eventos a fim de desenvolver } \\
\text { conjuntamente estratégias de prevenção } \\
\text { de erros e promoção de uma cultura de } \\
\text { segurança. }\end{array}$ \\
\hline
\end{tabular}

Fonte: Dados da pesquisa, 2018. 
Foi realizada leitura minuciosa e categorização dos resultados apresentados, com o propósito de melhor descrever e sintetizar os resultados obtidos da temática proposta. Os achados foram apresentados e discutidos de forma descritiva.

\section{DISCUSSÃO}

\section{Desafios}

A pesquisa de Hoefel HHK et al. (2017) relatou que a segurança do paciente é uma grande preocupação mundial destacada ultimamente. Esse estudo contribui para diversas ações preventivas a fim de impedir erros de medicação, ao levantar considerações importantes. Pautaram-se questões de tecnologia, atitudes e erros humanos, questões socio técnicas, cultura e resiliência.

Em seu estudo, Tomazoni A et al. (2017) descobriram que a segurança do paciente é afetada por rotinas cansativas de trabalho que podem colocar o paciente em diversas situações de perigo. Com isso, os profissionais sentem-se sobrecarregados ao tentar oferecer um cuidado seguro e sem danos. Alguns participantes deste estudo relataram que assegurar a segurança do paciente não é prioridade. Uma escolha para a melhoria de partilha das ocorrências de erros no cuidado sugere que os membros da equipe devem comunicar entre si erros e situações de perigo à segurança do paciente. A partir disso, seria executável propor a elaboração conjunta de algumas soluções por meio da promoção de uma educação permanente efetiva entre todos os componentes da equipe e gestores para que as ocorrências sejam evitadas e os riscos diminuídos.

O estudo de Novaretti MCZ et al. (2015) discutiu aspectos facilitadores da incidência de erros na administração de medicamentos pela equipe de Enfermagem em Unidade de Terapia Intensiva (UTI). Verificou-se que neste setor a cultura de segurança do paciente faz-se ainda mais necessária, pois nele os pacientes encontram-se especialmente vulneráveis, demandam decisões emergenciais e críticas, utilizam extenso arsenal medicamento e submetem-se a procedimentos invasivos devido a condições de saúde graves e instáveis. A cultura de segurança pode ser prejudicada pela sobrecarga de trabalho da equipe prestadora de assistência.

A equipe de Enfermagem que trabalha em UTI deve estar preparada para os desafios rotineiros, imbricados à complexidade da assistência e às cobranças dos pacientes e familiares, equipe multiprofissional e instituição. Na presença do erro na administração do medicamento, a equipe poderá sentir-se frustrada e envergonhada, o que dificulta a notificação e abordagem sobre o assunto (DUARTE SCM et al., 2015).

Um dos erros bastante comuns se encontra na diluição do medicamento, correspondendo a um malefício constante e até por vezes mortal, principalmente quando os pacientes se encontram bastantes vulneráveis e polimedicados. A diluição do medicamento exige atenção especial, pois cada fármaco possui suas especificidades no momento de preparo, nesse sentido, sendo relevante a verificação de qual solvente a medicação necessita, o recipiente, bem como tempo gasto até o fim do processo (MARINI DC et al., 2016).

É sabido que os profissionais de Enfermagem são humanos e passíveis de erros e que infelizmente incidentes devem ser esperados, em qualquer lugar, até mesmo nas excelentes organizações promotoras de saúde. Com isso, os gestores de saúde devem estar atentos ao controle de recursos humanos e financeiro, a frequência e a devida utilização de normas institucionais, ou seja, o estado do meio em que se trabalha, a apuração das sobrecargas de trabalho dos profissionais e as adequadas condições de funcionamento de todos equipamentos. Os fatores das causas de falhas associadas à estrutura física e déficits de materiais se especificam às condições do ambiente como uma grande desorganização e diversas interrupções frequentes durante procedimentos assistenciais, também levando em conta a falta de humanização por parte de alguns profissionais (SILVA AEBC e CASSIANI SHB, 2013).

\section{Perspectivas}

Para a saúde dos pacientes internados, a medicação é um cuidado fundamental, constituindo-se um elemento central na organização do trabalho da equipe de Enfermagem. Os riscos relacionados a segurança 
dos paciente e o grande ônus financeiro resultado dos custos com a medicação chamam atenção dos sistemas de saúde, de modo que se torna crucial um melhor entendimento sobre a cultura de segurança, a fim de destacar a Enfermagem e suas perspectivas na prevenção de agravos por erros durante a medicação (MAGALHÃES AMM, et al., 2015).

A Enfermagem empreende um valoroso papel no processo de medicação e, principalmente, na etapa final desse processamento, a administração. Esses profissionais são responsáveis pelo preparo, checagem de informações (data, horário, nome do paciente, via de administração, dose e forma do medicamento), administração, análise do efeito da droga administrada e a documentação de incumbência realizada. Ademais, a equipe é frequentemente descrita como a que está sempre presente e, portanto, responsáveis pela prevenção de erros de medicação no último estágio desse processo: a administração. Nesse sentido, torna-se importante a aplicação de intervenções como treinamentos para os profissionais sobre administração segura de medicamentos, reconhecimento e notificação na ocorrência de erros/eventos adversos e instalação de serviços de planejamento com sistema de rastreabilidade e prevenção de erros na farmácia. Todas essas sugestões podem ser estratégias iniciais em busca de maior segurança ao paciente (MARINI DC, et al., 2016).

O estudo de Magalhães AMM et al. (2015) projetou como intervenção positiva a implementação dos dispensários eletrônicos de medicamentos, recursos que garantem maior precisão e segurança no processamento. Contudo, os autores salientam que, apesar da avaliação positiva de seus usuários, esta ferramenta não extingue eventuais falhas no processamento.

Nas UTIs, por exemplo, a equipe de Enfermagem pode contribuir para a redução dos erros ao dispor de maior conhecimento sobre os aparatos tecnológicos, pois estes, muitas vezes, consistem em meios de comunicação entre o doente crítico e a equipe. Além disso, atividades de supervisão e auditoria devem ser encorajadas como forma de prevenir erros e garantir a administração segura de medicamentos. Importante ressaltar que nas UTIs há um grande volume de fármacos administrados diariamente, principalmente na via endovenosa. Erros por essa via tendem a ser irreversíveis e difícil manejo (MOREIRA APA, et al., 2017).

Hoefel HHK, et al., (2017) afirmaram que segurança do paciente se tornou mais efetiva, sobretudo com a difusão da cultura do compartilhamento de incidentes, o que facilitou a maneira de identificação dos casos e notificações voluntárias dos profissionais, permitindo buscar novas medidas que sejam capazes de reduzir riscos. Os autores também defendem que a cultura da culpabilização e julgamento deve ser abandonada.

Vale destacar que o papel da equipe de Enfermagem em prol da administração segura de medicamentos deve ser realizado sob a ótica da humanística, na qual o profissional está sensível a demanda do paciente e preocupado com a prevenção de erros. Desse modo, evita-se a prestação de uma assistência robotizada e mecânica, onde pacientes são vistos como seres admitidos, transferidos, que recebem alta ou vão a óbito (REIS LS, et al., 2013; CHERNICHARO IM, et al., 2013).

\section{CONSIDERAÇÕES FINAIS}

A administração que foge o preconizado pela segurança do paciente infelizmente ainda é problema presente em instituições hospitalares nacionais e internacionais, de modo que esforços têm sido feitos por meio de portarias nacionais que primam pela segurança do paciente. A equipe de Enfermagem possui um papel crucial na prevenção de iatrogenias medicamentosas, pois são os profissionais responsáveis desde o preparo à administração, estando presentes, assim, na última barreira que pode impedir o erro. Entretanto, esses profissionais enfrentam desafios como sobrecarga de trabalho, falhas associadas à estrutura física $e$ material, condições do ambiente (desorganização) e interrupções frequentes ao serem responsáveis por muitos procedimentos e pacientes. Todavia, há perspectivas promissoras. A capacitação e treinamentos podem ser estratégias viáveis à mudança desse cenário problemático, estimulando o profissional de Enfermagem à realização de boas práticas na administração de medicamentos. Outro recurso útil poderia ser a extinção da cultura de culpa por erros e incidentes cometidos, ainda presente em muitos serviços de saúde.

REAS/EJCH | Vol.Sup.25 | e452 | DOI: https://doi.org/10.25248/reas.e452.2019 Página 7 de 8 


\section{REFERÊNCIAS}

1. CAPUCHO HC et al. Segurança do paciente: comparação entre notificações voluntárias manuscritas e informatizadas sobre incidentes em saúde. Revista Gaúcha de Enfermagem, 2013; 34(1): 164-172.

2. CHERNICHARO IM et al. Caracterização do termo humanização na assistência por profissionais de enfermagem. Escola Anna Nery, 2014; 18(1): 156-162.

3. COUTO RC et al. Anuário da segurança assistencial hospitalar no Brasil. Belo Horizonte: Instituto de Estudos de Saúde Suplementar, 2017.

4. DUARTE SCM et al. O erro humano no cotidiano da assistência de enfermagem em terapia intensiva. Revista Latino-Americana de Enfermagem, 2015; 23(6): 1074-1081.

5. FASSARELLA CS et al. Segurança do paciente no ambiente hospitalar: os avanços na prevenção de eventos adversos no sistema de medicação. Revista de Cuidados em Saúde, 2013; 7(1): 1-8.

6. HOEFEL HHK et al. Incidentes de segurança ocorridos com pacientes durante o cuidado de enfermagem. Revista de Epidemiologia e Controle de Infecção, 2017; 7(3): 174-180.

7. KEERS RN et al. Causes of medication administration errors in hospitals: a systematic review of quantitative and qualitative evidence. Drug Safety, 2013; 36(11): 1045-1067.

8. KOHN LT et al. To err is human: Building a Safer Health System. Committee on Quality of Health Care in America; Institute of Medicine. Washington: National Academy Press, 2000.

9. JHA AK et al. The global burden of unsafe medical care: analytic modelling of observational studies. BMJ Quality \& Safety, 2013; 22(10): 809-815.

10. LEUFER T, CLEARY-HOLDFORTH J. Let's do no harm: medication errors in nursing: part 1. Nurse Education in Practice, 2013; 13(3): 213-216.

11. LIMA JÚNIOR AJ, GERHARDT LM. Desafio global da organização mundial da saúde: redução de danos associados à administração de medicamentos. Revista Contexto \& Saúde, 2017; 17(32): 1-4.

12. MAGALHÃES AMM et al. Processos de medicação, carga de trabalho e a segurança do paciente em unidades de internação. Revista da Escola de Enfermagem da USP, 2015; 49(Esp): 43-50.

13. MAIA CS et al. Notificações de eventos adversos relacionados com a assistência à saúde que levaram a óbitos no Brasil, 2014-2016. Revista Epidemiologia e Serviços de Saúde, 2018; 27(2): e2017320.

14. MARINI DC et al. Avaliação dos erros de diluição de medicamentos de administração intravenosa em ambiente hospitalar para o desenvolvimento de um guia de diluição e administração dos mesmos. Infarma, 2016; 28(2): 81-89.

15. MCLENNAN SR et al. Nurses' perspectives regarding the disclosure of errors to patients: a qualitative study. International Journal of Nursing Studies, 2016; 54: 16-22.

16. MOREIRA APA et al. Use of technologies in intravenous therapy: contributions to a safer practice. Revista Brasileira de Enfermagem, 2017; 70(3): 595-601.

17. NOVARETTI MCZ et al. Sobrecarga de trabalho da Enfermagem e incidentes e eventos adversos em pacientes internados em UTI. Revista Brasileira de Enfermagem, 2014; 67(5): 692-699.

18. REIS LS et al. Percepção da equipe de enfermagem sobre humanização em unidade de tratamento intensivo neonatal e pediátrica. Revista Gaúcha de Enfermagem, 2013; 34(2): 118-124.

19. SILVA ACA et al. A segurança do paciente em âmbito hospitalar: revisão integrativa da literatura. Cogitare Enfermagem, 2016; 21(1): 1-9.

20. SILVA AEBC, CASSIANI SHB. Análise prospectiva de risco do processo de administração de medicamentos anti-infecciosos. Revista Latino-Americana de Enfermagem, 2013; 21(Esp): 1-9.

21. SOARES CB et al. Revisão integrativa: conceitos e métodos utilizados na enfermagem. Revista da Escola de Enfermagem da USP, 2014; 48(2): 335-45.

22. SOUZA MT et al. Revisão integrativa: o que é e como fazer. Einstein, 2010; 8(1pt1): 102-106.

23. TOMAZONI A et al. Segurança do paciente na percepção da enfermagem e medicina em unidades de terapia intensiva neonatal. Revista Gaúcha de Enfermagem, 2017: 38(1): e64996. 